\title{
Emergencia hídrica, ambiental y climática. Elementos para su configuración y relación en el Estado de Derecho
}

Emergency in water, environment and climate. Configuration and relationships within the rule of law

Emergência bídrica, ambiental e climática. elementos para sua configuração e relação no Estado de Direito

Urgence bydrique, environnementale et climatique. Elements pour sa configuration et relation dans un Etat de Droit

水环境和气候紧急情况。法治中其构成和关系的要素

Liber Martín ${ }^{1}$

Universidad Nacional de Cuyo - Argentina

Revista Derechos en Acción ISSN 2525-1678/ e-ISSN 2525-1686

Año 5/No 14, Verano 2019-2020 (21 diciembre a 20 marzo), 224-253

DOI: https://doi.org/10.24215/25251678e358

ORCID: https://orcid.org/0000-0002-8827-6479

Recibido: 23/12/2019

Aprobado: 10/03/2020

\footnotetext{
1 Abogado (2003), Doctor en Derecho por la Universidad de Zaragoza (2009) y por la UNCuyo (2009). Miembro de la carrera del investigador científico del Conicet e investigador categoría IV (Com. Nac. de Acreditaciones). Profesor de Derecho Administrativo II de la UNCuyo y de Derecho Ambiental y de Recursos Naturales de las Universidades Nacional de Cuyo y de Mendoza. Ha publicado Derecho de aguas. Estudio sobre el uso y dominio de las aguas públicas (Abeledo-Perrot); en coautoría, El derecho humano al agua. Particularidades de su reconocimiento, evolución y ejercicio (Abeledo-Perrot) y co-dirigido Régimen jurídico de la evaluación de impacto ambiental (Lajouane).
} 
Resumen: El artículo estudia, primero la emergencia hídrica fundada en la sequía como fenómeno jurídico, excepcional y dispositivo de gobierno que, utilizado en el marco de un Estado de Derecho, plantea una serie de problemas fundamentales. Desde la óptica de la excepción se analiza cómo las normas de emergencia por sequía exceptúan el principio de legalidad, de competencia, las formas de contratación, gasto y subsidios, el otorgamiento de derechos, tanto como los criterios de distribución y los caudales ecológicos preestablecidos. En segundo lugar, el desarrollo se proyecta al ámbito ambiental y del cambio climático luego de lo cual se abordan los tres elementos claves para la configuración de la emergencia: la determinación científica de la situación de emergencia y su duración o plazo. Finalmente se advierte sobre los riesgos que esa emergencia, como principal reacción y creciente respuesta normativa frente a la crisis hídrica, ambiental y climática contemporánea, puede plantear al tornarse permanente puntualizando diferencias, relaciones e implicancias recíprocas en los tres ámbitos de referencia a los que se dirigen las conclusiones.

Palabras clave: Emergencia hídrica - emergencia climática - estado de excepción - cambio climático

Abstract: From a comparative approach the article first studies water emergency founded on drought as a legal phenomenon, exception and government device that, used in the framework of a rule of law, raises a number of fundamental problems. From the exception point of view, it analyzes how emergency rules for drought except the principle of legality, competition, contracting and spending forms and subsidies, water rights granting, as well as water distribution criteria and ecological flows. Secondly, the environmental and climate emergency are addressed while identifying the three key elements for the emergency configuration: the scientific and objective determination of the emergency situation; its duration or term and the possible configuration of a permanent emergency or state of emergency. Finally, it warns about the risks that this emergency, as the main reaction and growing regulatory response to the contemporary water, environmental and climate crisis, may pose by becoming permanent, while it specify differences, relationships and reciprocal implications in the three areas of reference.

Keywords: Water emergency - climate emergency - state of exception - climate change 
Resumo: 0 artigo estuda, primeiro a emergência hídrica fundada na seca como fenômeno legal excepcional e dispositivo governamental que, usado no âmbito do Estado de Direito, apresenta uma série de problemas fundamentais. Dede a perspectiva da exceção analisa-se como os regulamentos de emergência por seca exceptuam o princípio de legalidade, de concorrência, as formas de contratação, gastos e subsídios, a concessão de direitos, tanto com os critérios de distribuição e os fluxos ecológicos pré-estabelecidos. Em segundo lugar, o desenvolvimento é projetado ao meio ambiente e ao mudanças climáticas, após o qual são abordados os três principais elementos para a configuração da emergência: a determinação científica da situação de emergência e sua duração ou prazo. Finalmente alerta-se sobre os riscos que essa emergência, como principal reação e crescente resposta regulatória frente a crise hídrica, ambiental e climática contemporânea, pode gerar tornando-se permanente, destacando diferenças, relações e implicações recíprocas nas três áreas de referência às quais eles dirigem as conclusões.

Palavras-chave: Emergência hídrica - emergência climática - estado de exceção - câmbio climático.

Résumé: L'article étudie, tout d'abord, I'urgence hydrique fondée sur la sécheresse en tant que phénomène juridique exceptionnel et dispositif gouvernemental qui, utilisé dans le cadre de l'état de droit, plante une série de problèmes fondamentaux. A partir du cas d'exception, il analyse comment les normes d'urgence en cas de sécheresse permettent de faire exception au principe de légalité, de concurrence, aux formes de contrat, aux dépenses et subventions, à l'octroi de droits, ainsi qu'aux critères de distribution et aux flux écologiques préétablis. Deuxièmement, il traite des questions environnementales et du changement climatique, après quoi les trois éléments clés pour la configuration de la situation d'urgence sont abordés: la caractérisation scientifique de la situation d'urgence, sa durée ou son terme et la possible configuration d'une urgence ou d'un état d'urgence permanant. Enfin, il met en garde sur les risques que cet état urgence, comme principale réaction et réponse normative croissante face à la crise contemporaine de l'eau, de l'environnement et du climat, peut poser en devenant permanent, mettant en évidence les différences, les relations et les implications réciproques dans les trois domaines de référence.

Mot-clés: urgence hydrique - urgence climatique - état d'exception changement climatique 
摘要：本文首先研究以干旱作为一种特殊的法律现象而建立的水 紧急状况, 以及在法治框架内使用的政府手段带来了一系列基本问 题。从例外的角度分析了干旱应急条例如何免除合法性, 竞争, 合同 形式，支出和补贴，权利授予，分配标准和预先建立的生态流量原 则。其次, 预测环境和气候变化的发展, 然后讨论紧急情况配置的三 个关键要素: 科学确定紧急情况及其持续时间或期限。最后, 需要警 告的是, 这种紧急情况作为对当代水, 环境和气候危机的主要反应 和日益增加的监管反应，可能会变成永久性，并突出三个参考领域 之间的差异, 关系和相互影响, 可能带来的风险。他们指导结论。

关键词: 水紧急情况, 气候紧急情况, 紧急状态, 气候变化

El cambio climático tiene entre sus ámbitos principales de impacto a los recursos hídricos, tanto por su relación directa y esencial con la vida del ser humano, como por sus potenciales efectos destructivos o dañosos del ambiente donde habita. Sequía, inundación, subida del nivel del mar, retracción de glaciares, huracanes, derretimiento de polos, entre otros fenómenos, verán incrementada su intensidad y frecuencia como consecuencia del cambio climático.

Aunque operan en planos diversos la emergencia hídrica se vincula directamente con emergencia ambiental, y estas dos a su vez con la climática, de manera tal que entre ellas existen recíprocas relaciones de implicación tanto a nivel causal, como legal y argumentativo que es preciso develar.

El estado de emergencia, emergencia hídrica, o denominación equivalente (según el país de que se trate), es el concepto y estado usualmente declarado por las autoridades públicas ante situaciones de sequía o inundación (entendidas como catástrofes) que demandan actuaciones urgentes para hacer frente a una situación crítica de carácter excepcional. Sin embargo, las sequías o inundaciones son recurrentes y resultan características regulares de algunos climas, aunque muchas veces se produzcan o intensifiquen como consecuencia directa de la previa intervención humana en los ecosistemas que incrementan su 
vulnerabilidad y disminuyen su resiliencia frente a cualquier variación o modificación.

Las sequías e inundaciones no se deben entonces al cambio climático pero tal como lo constatan los documentos y estudios científicos disponibles están y van a verse paulatinamente incrementadas en su frecuencia e intensidad por este fenómeno ${ }^{2}$. Esto supone sequías o inundaciones más largas o pronunciadas, cambios en el régimen y parámetros de la precipitación o el escurrimiento, mayor evapotranspiración, y, en definitiva, cambios en la disponibilidad espacial y temporal hasta ahora considerada "normal" de recursos hídricos para una región, lugar y época determinados.

De allí también, la creciente preocupación por la seguridad hídrica, energética, alimentaria que subyace a enfoques como el del nexo agua-energía-alimentación-cambio climático. Lo que en definitiva ocurre, es que el cambio drástico en los parámetros temporales y espaciales de distribución de los recursos hídricos somete a prueba la capacidad efectiva de los sistemas de gestión, las instituciones y dentro de ellos, muy especialmente, al derecho y los instrumentos jurídicos ${ }^{3}$.

En este artículo se busca analizar sucintamente primero en el ámbito de los recursos hídricos, la recepción del instituto de la emergencia (I) y la normativa de emergencia propiamente dicha para analizar su contenido desde la óptica de la excepción (II). Proyectando ese desarrollo al ámbito ambiental y del cambio climático (III), se abordan los tres elementos claves para la configuración de la emergencia: la determinación científica y objetiva de la situación de emergencia y la duración o plazo de la emergencia (VI), luego de cual se presentan algunas conclusiones $(\mathrm{V})$.

2 EMBID IRUJO, Antonio (Dir.) (2018), Sequía e inundación como fenómenos hidrológicos extremos, Cizur Menor, Thomson Reuters Aranzadi.

3 Ver EMBID, A. MARTín, L. (2017), El nexo entre el agua, la energía y la alimentación en América Latina y el Caribe. Planificación, marco normativo e identificación de interconexiones prioritarias. SRNI, Santiago de Chile, CEPAL, 70 pp. 


\section{La emergencia en el derecho de aguas comparado}

Los mecanismos e institutos contenidos en las leyes de aguas o el derecho de aguas clásico para lidiar con la escasez, incluso extraordinaria (régimen de prioridades, cláusula sin perjuicio de terceros, jerarquía de derechos, turnos, etc.), no sólo en su diseño estructural, sino sobre todo en su aplicación práctica, muestran signos evidentes de obsolescencia, ineficacia y por tanto crisis ${ }^{4}$. En esa línea, como respuesta a ese fenómeno extendido y por causas diferentes, varias legislaciones han comenzado a incorporar o modificar recientemente disposiciones para la regulación de declaraciones y poderes de emergencia durante la sequía. Este es el caso de Chile, California y España por ejemplo.

El Código de Aguas (en adelante CAg.) de Chile, por ejemplo, dispone que el Presidente de la República, a petición o con informe de la Dirección General de Aguas, podrá, en épocas de extraordinaria sequía, declarar zonas de escasez por períodos máximos de seis meses, no prorrogables. Para lo cual la Dirección General de Aguas calificará, previamente, mediante resolución, las épocas de sequía que revistan el carácter de extraordinarias (art. 314 CAg). A continuación la disposición desarrolla el detalle las facultades de emergencia de la DGA, que son objeto del apartado siguiente, incrementándolas.

El Código de Aguas de California, dedica la sección 1058.5 íntegramente a las regulaciones de emergencia por sequía habilitando a la Autoridad de Aguas a dictar regulación de emergencia en dos supuestos. El primero, sin intervención de ningún otro poder, cuando se esté “...en condiciones existentes o amenazantes, en un año críticamente seco precedido por 2 o más años consecutivos por debajo de lo normal, secos o

\footnotetext{
4 MARTIN Liber (2010), Derecho de Aguas. Estudio sobre el uso y dominio de las aguas públicas, Buenos Aires, Abeledo Perrot; MARTín, Liber y PINTO, Mauricio (2015). Escasez extraordinaria y derecho de aguas en: Revista de Derecho Administrativo Económico N N 20 , Santiago, PUCC.
} 
críticamente secos". El segundo supuesto es durante la vigencia de un estado de emergencia oficialmente declarado por el Gobernador en el marco de lo dispuesto por la Ley de Servicios de Emergencia de California por razones de sequía.

La regulación de California, como la de Chile, pone límites a la duración de las regulaciones de emergencia. Sin embargo, los mismos han resultado relativos o parcialmente ineficaces frente a la prolongación de la sequía o una disminución progresiva de las precipitaciones como de hecho se prevé a futuro. Y ello porque los dos tipos de límites, tanto el relativo a las condiciones objetivas para la utilización de los poderes, como el establecimiento de un plazo fijo preestablecido o determinado por ley, presentan limitaciones evidentes en la práctica.

El primer límite no funciona frente a años críticamente secos que podrían ser cada vez más frecuentes como de hecho se prevé, con lo que los poderes de emergencia se ejercerán cada vez más a menudo. Nada impide, por otra parte, que los poderes se ejerzan alternadamente dentro del primero de los supuestos en algunos períodos y en el marco del segundo, en otros, para cuyo dictado no se exige más que la declaración oficial del estado de emergencia por parte del Gobernador.

El segundo límite refiere al plazo de vigencia de las regulaciones, pero lógicamente carece de toda consistencia por definición. El último inciso establece que las disposiciones de emergencia tendrán una vigencia de hasta 270 días, pero que puede ser renovado por la misma autoridad de aguas si las condiciones de sequía persisten. Con lo cual resulta de toda lógica, que las regulaciones de emergencia durarán lo que dure la sequía.

El Código Chileno pone el límite de 6 meses, estableciendo su carácter de improrrogable, planteando una serie de interrogantes: ¿Puede establecerse la duración de una sequía con antelación? ¿Qué sucede si la sequía continúa? Con mayor o menor respeto a la formalidad el régimen de emergencia continuará o se prorrogará de alguna manera? ¿Se volverá al régimen 
de normalidad justo cuando la situación sea aún más grave y acuciante por su prolongación más allá de las previsiones del legislador?

Esta contradicción parte del presupuesto erróneo de considerar la sequía como una catástrofe impredecible o evento de carácter extraordinario, dado que justamente cuando más se puede hacer para paliar los efectos de una sequía es sobre todo antes, no durante la misma como lo demuestra el enfoque preventivo adoptado en el caso español o el énfasis en los sistemas de alertas e indicadores que permitan su pronóstico temprano.

Es evidente que bajo esta regulación existe un riesgo cierto de que la emergencia se utilice cada vez con mayor frecuencia, cuando no prolongue sus efectos más allá de la existencia formal de la sequía. La evolución de la recurrencia con la que se utilizan estos poderes de emergencia debe ser seguida muy de cerca para advertir cuando, justamente, la misma ha dejado de ser excepcional para transformarse en permanente.

El texto Refundido de la Ley de Aguas Español (TRLA), por su parte, regula las situaciones excepcionales estableciendo que: "En circunstancias de sequías extraordinarias, de sobreexplotación grave de acuíferos, o en similares estados de necesidad, urgencia o concurrencia de situaciones anómalas o excepcionales, el Gobierno, mediante Decreto acordado en Consejo de Ministros, oído el organismo de cuenca, podrá adoptar, para la superación de dichas situaciones, las medidas que sean precisas en relación con la utilización del dominio público hidráulico, aun cuando hubiese sido objeto de concesión. La aprobación de dichas medidas llevará implícita la declaración de utilidad pública de las obras, sondeos y estudios necesarios para desarrollarlos, a efectos de la ocupación temporal y expropiación forzosa de bienes y derechos, así como la de urgente necesidad de la ocupación" (Art 58 TRLA).

Sin embargo, el régimen sustancial general de la sequía del derecho español de aguas encuentra otras disposiciones, como el art. 27 de la Ley 10/2001, de 5 de julio, del Plan Hidrológico 
Nacional. A diferencia de los anteriores, este pone el énfasis en la actuación previa de los poderes públicos, mediante primero, el establecimiento de un sistema global de indicadores hidrológicos que permita prever estas situaciones y sirva de referencia general a los Organismos de cuenca para la declaración formal de situaciones de alerta y eventual sequía. En segundo lugar, ordena un proceso especial de planificación para la sequía con instrumentos diferenciados para la cuenca y los abastecimientos urbanos.

Al margen de otras cuestiones, este enfoque con énfasis en la planificación evidencia un salto cualitativo respecto del tratamiento meramente reactivo frente a la sequía que muestran otros casos que sólo refieren un marco para el dictado de normativa y medidas de emergencia, sin perjuicio de las críticas de que pueden ser pasibles los Planes Especiales de Sequías (PES) ${ }^{5}$.

\section{Las normas de emergencia por escasez o sequia. Caracterización de su contenido de excepción}

\section{Excepción al principio de legalidad. Forma de la normativa de emergencia}

Un aspecto crucial pero sobre el que poco se repara es la naturaleza jurídica o el rango de la normativa de emergencia. Esto es que la regulación de emergencia, a pesar de su fuerte contenido restrictivo o excepcionador, es instaurada normalmente por decretos, reglamentos o actos administrativos, y rara vez por leyes, quebrando el principio de legalidad.

En Chile la excepción a la aplicación del Código de Aguas, va a ser decidida conforme la previsión del mismo Código por Decreto presidencial a petición o con informe de la Dirección General de Aguas. Si bien se trata de una facultad delegada por

\footnotetext{
5 BRUFAO CURIEL, Pedro (2012). El régimen jurídico de las sequías: crítica a la regulación extraordinaria y urgente de un fenómeno natural y cíclico propio del clima. En: Revista de administración pública 187, pp. 199-239; EMBID IRUJO, Antonio (2018). Sequía e inundación como fenómenos hidrológicos extremos, Thomson Reuters Aranzadi.
} 
ley, es el Poder Ejecutivo el que en definitiva decide la excepción a la norma a través de la suspensión de la aplicación del Código en los aspectos que será reemplazado por las disposiciones de la DGA (art. 314 del CAg Chile).

En el caso español, si bien el TRLA prescribe que la regulación de circunstancias excepcionales debe adoptar la forma de Decreto acordado en Consejo de Ministros con audiencia del organismo de cuenca (art. 58 TRLA), el Gobierno ha utilizado la figura del Real Decreto tanto como al Real Decreto Ley para la regulación de la sequía 2005-2008. Algunos de ellos, de hecho con efecto para todo el territorio (RD-Ley 10/2005; RDLey 15/2005; RD 287/2006), otros con respecto a cuencas o determinados grupos de cuencas en particular ${ }^{6}$.

La experiencia española es relevante puesto que es de los pocos casos en los que la jurisprudencia ha tenido oportunidad de controlar judicialmente la normativa de emergencia con fundamento en la sequía, llegando incluso a declarar su nulidad, aunque precisamente por vicios de procedimiento y no por la puesta en cuestión de la situación fáctica misma, es decir la existencia o no de sequía ${ }^{7}$.

En California la normativa de emergencia para paliar los efectos de la sequía puede tener diversas fuentes. Puede, de hecho, tratarse de normas legislativas federales o estatales, específicas o generales como algunas modificaciones al Código de Aguas. Sin embargo, la normativa de emergencia propiamente dicha tiene normalmente el rango de Executive Order, comparable al

6 Vid. BRUFAO CURIEL, Pedro (2012). El régimen jurídico de las sequías: crítica a la regulación extraordinaria y urgente de un fenómeno natural y cíclico propio del clima, RAP, 187, pp. 199-239; URQUIJO, Julia, et al, (2005) Drought and exceptional laws in Spain: the official water discourse, en International Environmental Agreements. Politics. Law and Economics, Springer, 2005.

7 Este es el caso del RD 1265/2005, de 21 de octubre o el RD 1419/2005, de 25 de noviembre declarados nulos por la falta de audiencia de las confederaciones hidrográficas y sus juntas de gobierno, entre otras omisiones, y respectivamente por las sentencias del Tribunal Supremo Español STS, Sala 3. a , Sección 5. ', de 20 de enero de 2009 y STS, Sala 3. ${ }^{a}$, Sección $5 .{ }^{a}$, de 24 de noviembre de 2009 , respectivamente. 
decreto, cuando es declarada por el Gobernador de California o de un reglamento cuando lo es por la Autoridad de Agua, que es en definitiva, donde se va encontrar el régimen sustancial de la emergencia, conforme los poderes antes referidos.

\section{Excepción a la competencia. Incremento de facultades, mayor discrecionalidad y reducción de controles}

El aumento de las competencias o poderes y de la discrecionalidad de la autoridad administrativa, junto con la reducción o suspensión de los mecanismos de control, es quizás la característica común más fácil de apreciar sobre el contenido de la regulación de emergencia en todos los casos revisados.

Es notable, por ejemplo, la amplitud de poderes que conceden a la administración incluso el CAg. chileno, durante los periodos de escasez extraordinaria (art. 314). En la misma claramente puede observarse este rasgo que calza de manera muy ajustada en la hipótesis planteada confiriendo a la DGA las siguientes potestades:

a) Redistribuir las aguas, siempre que no haya acuerdo entre los usuarios para hacerlo. Y para ello podrá suspender tanto las atribuciones de la Junta de Vigilancia como el seccionamiento de los ríos.

b) Autorizar extracciones de aguas superficiales y subterráneas sin necesidad de constituir derechos de aprovechamiento / sin limitación de caudal ecológico.

c) Adoptar las medidas necesarias sin sujeción a las normas establecidas en el Título I del Libro Segundo del Código de Aguas (Procedimientos, constitución e derechos; bocatomas; cambio de fuente; etc.)

d) Dar efecto inmediato a sus resoluciones, sin perjuicio de la posterior toma de razón por la Contraloría General de la República.

Las amplias facultades conferidas por el Código y reproducidas por los decretos de declaración de escasez en los casos 
particulares por la DGA contrastan sin embargo con la perspectiva neoliberal del Código, pero se explican desde que no pertenecen a la redacción original sino que fueron incorporadas por la reforma de 2005, que efectúa varias modificaciones con relación a este punto ${ }^{8}$.

Esta tendencia es evidente en el caso de California donde también la emergencia por sequía se traduce en mayor poder, más discrecionalidad y menos controles para el actuar del State Board of Water Resources. El art. 1058.5 dispone en primer lugar que el Board podrá adoptar estas regulaciones por sí mismo cuando se esté "...en condiciones existentes o amenazantes, en un año críticamente seco precedido por 2 o más años consecutivos por debajo de lo normal, secos o críticamente secos" y sin mediar la declaración oficial de emergencia por parte del Gobernador. Es decir, y a diferencia de Chile por ejemplo, se trata de una habilitación legal permanente a un órgano de naturaleza administrativa que no requiere de declaración previa de emergencia alguna por parte de la Legislatura ni del Gobernador.

Estas regulaciones están además, expresamente exentas del control o revisión que efectúa la Oficina de Derecho Administrativo y finalmente, será el mismo Board quien defina la duración de su vigencia, que puede exceder por tanto el límite de los 270 días antes referido si la sequía persiste. Desaparece el control ex ante y queda por ver el marco de legalidad o constitucionalidad en el cual se procederá a su control judicial, donde la deferencia será previsiblemente mayor que en períodos de normalidad.

Esta regulación de emergencia alcanza los más variados aspectos con la finalidad de evitar el derroche, el uso no razonable, métodos de uso o de extracción irrazonables, para promover el ahorro y el reuso de agua, para requerir la interrupción

8 Ver: NOÉ SCHEINWALD, Paula (2015). Interfaz ciencia-política en la gestión de sequía en Chile, Academia.edu. 
de los derechos cuando no existe disponibilidad acorde las prioridades legales de los derechos, o en cumplimiento de cualquiera de los anteriores, para exigir la presentación de informes de extracción o uso o la preparación de los informes de monitoreo.

El Código de Aguas de California fue modificado en 2014 y como consecuencia de la sequía persistente desde 2012, amplió los poderes de la autoridad del agua en situaciones de emergencia, para asegurar los derechos de aguas, incrementando, entre otras cosas, las penas por extracciones ilegales durante la sequía (SB 104 adopted March 1, 2104).

Disparado el proceso de suspensión de las dotaciones con base en las prioridades legales que se analizan en el punto siguiente, la ineficacia y falta de acatamiento de los cortes por parte de los usuarios llevó a la introducción de nuevas modificaciones en el régimen de emergencia, básicamente consistentes en un incremento de las penas y en la eliminación de garantías y simplificación de los procedimientos de corta y aplicación de sanciones por su violación.

Las medidas de emergencia justifican también a menudo la excepción a las formas ordinarias de contratación de la administración (vg. Licitación pública) o a las autorizaciones requeridas para la construcción de obras, incompatibles con la celeridad requerida para contratar o aprobarlas en casos de urgencia.

Subsidios, ayudas extraordinarias o exenciones impositivas con carácter paliativo son también una clásica y típica respuesta a las situaciones consideradas de emergencia aunque, tal como se ha puesto en duda cualquier sequía no lo sea en sentido estricto en las regiones cuya legislación se analiza. Una enorme cantidad de fondos públicos son adjudicados y distribuidos en forma más o menos discrecional y al margen de los procedimientos normales, de adjudicación y control con fundamento en la emergencia por sequía, inundación, o la consiguiente declaración de emergencia hídrica, agropecuaria, sanitaria, etc. 


\section{Excepción a la forma de otorgamiento de derechos $y$ distribución de las aguas}

Si bien en situaciones de sequía extraordinaria no parece lógico asignar o constituir nuevos derechos, lo cierto es que la mayoría de las veces, bajo regímenes excepcionales como los analizados, se autorizan nuevos derechos o usos, particularmente en materia de aguas subterráneas y con el objeto de suplir la escasez de las superficiales.

El 314 de CAg. de Chile dispone que declarada la zona de escasez la DGA “...podrá autorizar extracciones de aguas superficiales o subterráneas desde cualquier punto sin necesidad de constituir derechos de aprovechamiento de aguas y sin la limitación del caudal ecológico mínimo establecido en el artículo 129 bis 1 . También podrá otorgar cualquiera de las autorizaciones señaladas en el Título I del Libro Segundo de este Código...". Con ello, la consecuente suspensión de la legislación: "Para los efectos señalados en los incisos anteriores, y lo dispuesto en el artículo siguiente, la DGA adoptará las medidas sin sujeción a las normas establecidas en el Título I del Libro Segundo de este Código".

La mayoría de los regímenes de emergencia recurren a las aguas subterráneas para suplir la escasez de las otras, incluso cuando esos acuíferos ya están comprometidos o sobreexplotados. Y esto puede hacerse excepcionando los procedimientos normales, para hacerlos más expeditos, a través de permisos "provisionales" o incluso rebajando los estándares ambientales o de calidad establecidos para determinados cuerpos de aguas?.

Esto es lo que ha ocurrido en la experiencia comparada de Chile y California donde se flexibilizan los criterios para la realización de perforaciones, con un carácter provisional que dudosamente respeten con posterioridad abriendo paso a su consolidación progresiva.

9 GREEN, Micah (2014). Keeping California's Thirst for Groundwater in Check, in McGeorge Law Review | Volume 46-2, pp. 346 y 347. 
Cuando existen mercados de aguas o centros de intercambio de derechos de aguas lo que ha hecho básicamente la regulación de emergencia es facilitar o acelerar las transferencias mediante la eliminación de requisitos de todo tipo para su aprobación.

Las formas de distribución de aguas en condiciones de sequía extrema presentan un desafío para el equilibrio del sistema, poniéndolo a prueba. Ello puede deberse a que la aplicación sin más de las reglas legales asociadas a la categoría de los derechos y basadas fundamentalmente en su antigüedad puede resultar problemática. De hecho el resultado de la aplicación estricta de estas categorías de derechos, no sólo puede resultar inequitativa o injusta sino y llegado el caso, incluso ineficiente o antieconómica.

Por ello se explica que en todos los casos y sin excepción, la regulación de emergencia establezca y ordene a la autoridad aplicar la distribución de las aguas en tiempo de sequía conforme la ley, pero al mismo tiempo, en una suerte de mandato que podría resultar contradictorio, confiere amplias atribuciones para asegurar determinados usos o para proveer a la mejor distribución de las aguas al margen de la ley, sin explicar cuál sería.

El CAg. de Chile confiere a la DGA amplias potestades para la redistribución de las aguas a falta de acuerdo entre los usuarios para hacerlo ${ }^{10}$. Una de las disposiciones más llamativas es la regulación de un específico supuesto de responsabilidad del Estado para el caso de que un usuario no reciba la dotación a la que tiene derecho (art. 314 CAg Chile).

En California, la distribución de aguas durante la sequía ha sido una cuestión mayor, habida cuenta la cantidad de derechos recortados y la urgencia por parte de la autoridad de definir esos criterios. Fue durante la sequía de 1976-1977 la primera vez que hubo que disponer cortas en base a prioridades legales antes del periodo de sequía 2012-2015. La complejidad deviene

\footnotetext{
10 VERGARA BLANCO, Alejandro (1999). El derecho de la seguía: la redistribución de aguas, en Revista de Derecho Administrativo Económico, Vol. I / N² 2, pp. 361-370.
} 
del intrincado sistema y las varias clases de derechos de aguas coexistentes en la actualidad donde se han desarrollado distintos modelos para hacer más eficiente esta distribución ${ }^{11}$.

El marco de lo anterior está dado por la Constitución de California que dispone como regla básica que los recursos hídricos del estado deben ser utilizados de manera beneficiosa, razonable y sin derrochar (art. X, section 2). La Corte Suprema de California ha sostenido por su parte que “...lo que puede considerarse un uso beneficioso razonable, mientras el agua disponible excede las necesidades, puede no ser razonable y beneficioso, en un área de gran escasez o necesidad. Lo que es un uso beneficioso en un momento, puede, por cambio en las condiciones, convertirse en un derroche o desperdicio de agua en un momento posterior" (Tulare Dist. v. Lindsay Strathmore Dist. (1935) 3 Cal.2d 489, 567.)

La Legislatura, por su parte, ha dispuesto expresamente en la Section 1011 del Código de aguas de California que las reducciones y ahorros dispuestos durante la sequía serán consideradas como uso equitativo y razonable durante la sequía y conforme el Art. X sección 2. Para favorecer el ahorro de agua, expresamente dispone que estas regulaciones temporarias de emergencia no podrán ser utilizadas en ningún procedimiento administrativo o judicial futuro como prueba o evidencia de derroche o irrazonabilidad en el uso de ningún usuario individual o proveedor sujeto a esta regulación y no podrá afectar ni limitar de cualquier manera los derechos sobre el agua ahorrada bajo el derecho aplicable, incluyendo sin limitación, el agua ahorrada en consonancia con la Sección 1011 del Código de aguas.

En Colorado, EEUU, el principio de legalidad primó sin embargo sobre criterios de eficiencia al ordenar el cegado de unas

11 HANAK, Ellen et al. (2014). Modernizing drought water allocations. http://californiawaterblog.com/2014/10/16/modernizing-drought-water-allocations/; LUND, Jay; LORD, Ben; FLEENOR, William y WILLIS, Ann. (2014): Drought Curtailment of Water Rights - Problems and Technical Solutions. Center for Watershed Sciences, UC Davis. Technical comments to the State Water Resources Control Board. 
cuatrocientas perforaciones nuevas y posteriores (junior rights) en favor de derechos de mayor antigüedad (senior rights), aun cuando los beneficios socio-económicos aportados por su explotación eran, como suele ocurrir, muy superiores a los derivados de la utilización de las antiguas ${ }^{12}$.

Como siguiendo un manual, las declaraciones de emergencia allí donde existen caudales ecológicos fijados, normalmente los sacrifican, rebajando los parámetros en pos de mantener el suministro de los usos convencionales. Esto es lo que va ocurrir con algunos matices en los casos de Chile, California y España.

El CAg. de Chile directamente establece que luego de la Declaración de Zonas de Escasez extraordinaria por parte del Presidente, la Dirección General de Aguas podrá autorizar extracciones de aguas superficiales o subterráneas...", "... sin la limitación del caudal ecológico mínimo..."(art. 314 CAg.).

El 25 de abril de 2014 el Gobernador de California proclamó la continuación del estado de emergencia por causa de sequía declarada el 17 de Enero de 2014. Entre varios agregados, suspendió los requerimientos de la California Environmental Quality Act (CEQA) para determinadas actividades incluidas las regulaciones de emergencia adoptadas bajo la sección 1058.5 del CAg. de California varias veces referida.

Esto es lo que ha ocurrido también en el caso español, por ejemplo, con el Real Decreto 1265/2005, de 21 de octubre, típica norma de emergencia, por el que entre otras medidas administrativas excepcionales se autoriza la reducción de los caudales mínimos para captaciones superficiales, la modificación de los criterios de prioridad y las dotaciones para los distintos usos. En particular, y entre otras, habilita a la Junta de Gobierno de las Confederaciones Hidrográficas a: a) Reducir las dotaciones en el suministro de agua que sean precisas para racionalizar la distribución de los recursos hídricos. b) Modificar los criterios de

12 HOWE, Charles (2008). Water Law and Economics: An Assessment of River Calls and the South Platte Well Shut-Down, 12 U. Denv. Water L. Rev. 181 (2008-2009). 
prioridad para la asignación de recursos a los distintos usos del agua, respetando en todo caso la supremacía del uso Consignado en el artículo 60.3.1. ${ }^{\circ}$ del texto refundido de la Ley de Aguas, aprobado por el RD Legislativo 1/2001, de 20 de julio; c) Modificar temporalmente y mediante resolución motivada los requerimientos medioambientales establecidos en los planes hidrológicos.

Lo interesante de este ejemplo, es el control judicial que sobre tal legislación de emergencia operó. En efecto, este RD va a ser declarado nulo con posterioridad por la STS, Sala 3, Sección 5 de 20 de enero de 2009, por no haber cumplido con el trámite debido de audiencia previa de las confederaciones hidrográficas ni de sus juntas de gobierno.

Incluso en la Directiva Marco de Aguas 2000/60, cuyo signo distintivo inequívoco es el de la protección de la calidad de las aguas, los eventos de sequía son sólo considerados -con excepción de una mención de la mitigación de la sequía entre los objetivos- a los efectos de calificarlos como "circunstancias excepcionales" que habilitan la derogación de los parámetros para alcanzar el buen estado ecológico de los cuerpos de aguas. El art. 4.6 de DMA contempla la sequía como causas naturales o de fuerza mayor en las que el deterioro temporal de las masas de aguas no importará un incumplimiento de la misma ${ }^{13}$. Claro que desde esta Directiva han transcurrido veinte años en los cuales la UE ha desarrollado una completa política sobre Sequías incluyendo el cambio de paradigma referido.

Con todo, es evidente que el sólo criterio de la antigüedad o prelación temporal de derechos consolidados hace más de un siglo, resulta insuficiente para distribuir las aguas en las situaciones actuales de escasez y sequía. Legalidad, prelación en el tiempo, equidad, eficiencia, derechos humanos y, preservación y prevención son todos principios de base constitucional

\footnotetext{
13 ROSSI, Giuseppe y CANCELLIERE, Antonio (2013). Managing drought risk in water supply systems in Europe: in Journal of Water Resources Development, 29:2, pp. 272-289, DOI: 10.1080/07900627.2012.713848.
} 
que tensionan fuertemente a la hora de redistribuir caudales en situaciones extremas. Demasiada complejidad para ser bien resuelta por una autoridad administrativa en el marco de la urgencia que impone la emergencia.

El análisis hasta aquí efectuado sugiere que es tan evidente que estas regulaciones de emergencia proveen mayor flexibilidad y capacidad de respuesta a los poderes públicos, como que muchas veces riñen con los elementales principios de legalidad, republicano, democrático, de responsabilidad, eficiencia, rendición de cuentas y de planificación de mediano y largo plazo que caracterizaron el Estado de Derecho hasta la actualidad.

La gran discrecionalidad administrativa habilitada en el marco de este tipo de emergencias parece estar limitada sólo tímidamente por el principio de razonabilidad -no más por el de legalidad-, y el control judicial se relaja y dificulta severamente cuando la misma es ejercida con fundamento en una declaración previa de emergencia hídrica con teórico fundamento en el carácter catastrófico, crítico o extraordinario de una sequía.

Dejar de considerar la sequía o inundación, como una catástrofe o fatalidad que sólo engendra medidas de carácter reactivo, excepcionales o de emergencia, implica asumir su naturaleza habitual que se condice mejor con un paradigma preventivo de gestión permanente de riesgos que en vez de comprometer o diluir, refuerza la función regulatoria.

\section{Proyeccion al ambiente y el cambio climático}

Agua, ambiente y cambio climático constituyen ámbitos diferentes aunque inescindiblemente vinculados y entrelazados. Lo son desde el punto de vista físico y también jurídico, corresponden a distintas escalas, tienen distinto marco normativo principios, políticas, planes, competencias y autoridades de aplicación. Dar cuenta de esas relaciones para superar esa fragmentación constituye un desafío frecuente que lleva a la necesidad de conectar o vincular las emergencias que puedan producirse en esos ámbitos cuando proceda. 
La emergencia ambiental ha sido utilizada en supuestos muy variados, desde calidad de aire (como en el caso de Ciudad de México) hasta contaminación de aguas u ordenamiento territorial. La ley 26168 de creación de la Autoridad de Cuenca Matanza Riachuelo hace referencia al artículo $2 \mathrm{k}$ de la ley 25675 en lo que puede considerarse un reconocimiento implícito de un estado de emergencia ambiental. De hecho, durante la ejecución del caso "Mendoza" (2008-2015), por ejemplo, la ACUMAR, utilizó en forma reiterada el argumento de la urgencia, para justificar la excepción al principio de licitación y contratar directamente, dando lugar a un escándalo con relación a la adjudicación de obras y fondos. Sin marco regulatorio específico, el Gobernador de Mendoza declaró el Estado de Emergencia Ambiental en la zona del Piedemonte del Área Metropolitana de Mendoza por 12 meses (Decreto $N^{\circ} 219,7 / 2 / 19$ ).

En cambio, la ley de Perú $\mathrm{N}^{\circ} 28804$ regula específicamente el procedimiento para declarar en Emergencia Ambiental una determinada área geográfica en caso de ocurrencia de algún daño ambiental súbito y significativo ocasionado par causas naturales, humanas a tecnológicas que deteriore el ambiente, ocasionando un problema de salud pública como consecuencia de la contaminacion del aire, el agua a el suelo; que amerite la acción inmediata sectorial a nivel local a regional (art. 1). En su art. $3^{\circ}$ se establece los criterios a tener en cuenta para la adopción de esa declaración ${ }^{14}$.

14 El Consejo Nacional del Ambiente, en coordinación con el Ministerio de Salud, aprueba los indicadores necesarios para efectos de la declaración en emergencia ambiental, teniendo en cuenta, los siguientes criterios: a) Nivel de concentración de contaminantes por encima de los Estándares de Calidad Ambiental o Límites Máximos Permisibles, aprobados en el país; 0 por las instituciones de derecho público internacional que sean aplicables o la Organización Mundial de la Salud (OMS), en forma referencial, cuando no existan estándares nacionales, verificados por la autoridad competente. b) Contaminación de la población y el ambiente por sustancias peligrosas por encima de los niveles que internacionalmente se considera aceptables para la salud humana, verificado por las autoridades de salud. c) Alto riesgo para poblaciones vulnerables. d) Ocurrencia de accidentes que generen emisión de vertimientos de sustancias peligrosas que, a pesar de no estar establecidas en la legislación nacional, están consideradas en los estándares o límites de instituciones u organismos internacionales, en 
Por su parte, el Secretario General de Naciones Unidas sintetizó la crisis del cambio climático de la siguiente forma: "This is an emergency and for emergency situations we need emergency action" (2010). Climate Emergency Declaration, por su parte, es un movimiento global que promueve la declaración formal política de emergencia climática y ambiental u ecológica por parte de los parlamentos y ha conseguido ya cerca de mil declaraciones en 2019, entre los que se encuentran Escocia, Reino Unido, Irlanda, Portugal, Canadá, Francia y Argentina, entre otros ${ }^{15}$. El último en hacerlo ha sido el propio parlamento Europeo con fecha 27 de noviembre de 2019, en cuyo debate estuvieron de hecho presentes, y en cuestión, los conceptos de "punto de no retorno", "emergencia", "urgencia" y "estado de excepción".

El Club de Roma presentó su Plan de Emergencia Climática al Parlamento Europeo en diciembre de 2018. Partiendo del presupuesto de que los compromisos derivados de Paris no resultan suficientes para conseguir el objetivo de evitar el aumento promedio de la temperatura global en $1,5 \mathrm{C}^{\circ}$, que entiende configura una situación de emergencia, el plan propone una serie medidas y acciones urgentes a adoptar por parte de los Estados.

Los casos mencionados de cambio climático revisten mero carácter declarativo, simbólico sin constituir por el momento una emergencia en sentido estricto o jurídico formal con el contenido suspensivo o excepcionador del ordenamiento jurídico utilizado para analizar la emergencia hídrica en este artículo. Sin embargo, si puede brindar una base importantísima para el paso siguiente, que es el proveer las bases de justificación y

\footnotetext{
forma referencial. e) Impactos a largo plazo en la salud humana. f) Ausencia de instrumentos de gestión ambiental que involucren planes de recuperación del área materia de la declaración. g) La protección de la vulnerabilidad y singularidad de los espacios naturales. (art. 3, Ley de Perú N²8804)

15 El Senado Argentino, por ejemplo, declaró la emergencia climática y ecológica global (DR-1079/19 Senado de la Nación) el mismo día que dio media sanción por unanimidad a la demorada Ley de Presupuestos Mínimos para la Adaptación y Mitigación del Cambio Climático, finalmente aprobada en noviembre de 2019.
} 
legitimación suficientes para lo que si pueden ser o convertirse en auténticas declaraciones y actuaciones de emergencia en materia climática, pero también ambiental o hídrica en tanto manifestación de la primera, con las características referidas en este artículo.

Como se adelantó en la introducción, este trabajo pone su énfasis en la forma jurídica de la respuesta que, en última instancia, debe dar el derecho nacional a los efectos o consecuencias que el cambio climático produce a nivel local, materializado en fenómenos hidrológicos extremos o crisis ambientales entre otras consecuencias.

La forma en que los poderes de emergencia en general o en particular se activan en cada país es muy diferente, tanto como el alcance, contenido y sustancia de los mismos, pero el fenómeno desde el punto de vista jurídico sustancial es similar. En el caso de Francia, por ejemplo, la declaración de "estado de urgencia" está regulada en una específica ley de 1955 que contempla los presupuestos generales, y la doctrina se ha explayado sobre las condiciones y posibilidades particulares de procedencia de un tal estado de urgencia ecológico o ambiental en sentido estricto, poniendo especial atención sobre lo que denominan la banalización del estado de urgencia, que no es otra cosa que la perpetuación del mismo o su falta de contenido ${ }^{16}$.

Estados Unidos, por su parte, tiene una muy desarrollada jurisprudencia y doctrina sobre el uso de poderes extraordinarios, orientados a garantizar la seguridad nacional, y tal como en el caso francés la doctrina ha reconocido y planteado seriamente la posibilidad de su uso con ocasión de la amenaza para la seguridad nacional que supone el cambio climático en su estadio actual, poniendo de manifiesto, entre otros muchos aspectos, las implicancias de retroceso que ello puede tener en términos institucionales y democráticos, tanto como el de

\footnotetext{
16 RRAPI, Patricia (2017), Changements climatique et urgence environnementale, I'hypothèse de l'état d'urgence écologique, en HALPÉRIN, Jean-Louis; et al (2017), L'état d'urgence: de I'exception à la banalisation, Paris, PUPN, p. 181 y ss.
} 
constituir una alternativa para sumar de una vez al país en esa lucha hasta la que ahora ha sido remiso ${ }^{17}$.

\section{Claves para la configuración de la emergencia en el Estado de Derecho}

El fenómeno de evolución y uso creciente de la emergencia hasta aquí descripto en el muy reducido ámbito de los recursos hídricos y, más concretamente, en relación a su escasez extraordinaria alcanza sin embargo a los más variados ámbitos del gobierno, presentando una serie de problemas y desafíos enormes para el derecho del siglo XXI. En efecto hay, como se verá continuación, muchas razones de peso para extenderlo a otros ámbitos como el ambiental o el propio del cambio climático incluso.

Existen distintas perspectivas para analizar el fenómeno en cuestión que naturalizan esta deriva, o por el contrario, la ponen en cuestión notando que compromete presupuestos constitucionales o principios capitales del Estado del Derecho como el propio principio democrático o republicano según la profundidad que el fenómeno alcance, el marco teórico y el alcance o interpretación que se le asigne.

Una vasta bibliografía se ha dedicado a tematizar estos enfoques que, según la denominación que se utilice, abarca la emergencia o el derecho de la emergencia, el estado de necesidad o urgencia, el uso de poderes excepcionales o extraordinarios, la distinción entre normal y anormal y el estado de excepción, entre otros, tanto en el ámbito propiamente jurídico como en el de las ciencias sociales. Y esto es así porque el fenómeno analizado es capital para el derecho y el estado de derecho, al punto de comprometer su propia existencia y consistencia ${ }^{18}$.

\footnotetext{
17 FARBER, Dan (2019), Declaring a Climate Change Emergency: A Citizen's Guide, https://legalplanet.org/2019/03/14/declaring-a-climate-change-emergency-a-citizens-guide/ [Cons.: 6/9/19].

Los medios periodísticos han reflejado igualmente este debate. https://thebulletin.org/2019/ 03/what-could-happen-if-a-democratic-president-declared-a-national-climate-emergency/; https://grist.org/article/trump-what-would-a-national-emergency-over-climate-change-look-like/
} 
Así, emergencia hídrica, emergencia ambiental y emergencia climática, por ejemplo, presentan una íntima y evidente conexión tanto por su posible implicación mutua como por el hecho de que tanto la hídrica como la ambiental, se conviertan o constituyan en realidad, la materialización concreta y tangible a nivel local de ese fenómeno de cambio climático que opera a nivel global. Sin pretensión de exhaustividad, la actuación de emergencia presenta unos problemas comunes que particularizándolos en el ámbito de los recursos hídricos, ambiental y climático pueden resumirse en los siguientes puntos:

\section{Determinación científica y objetiva de la situación de emergencia}

La cuestión capital a determinar consiste en determinar cuándo se está ante una situación de emergencia tal que justifique y requiera del marco de excepcionalidad jurídica. El caso de las múltiples definiciones de sequía tanto como su prórroga sistemática es muy elocuente al respecto y evidencia cierta inconsistencia de los mecanismos jurídicos excepcionales que la requieren como justificación como se vio.

La dificultad en materia climática está además dada porque partiendo del presupuesto de su origen antropogénico y las advertencias efectuadas desde hace al menos tres décadas por la ciencia en general y el IPCC en particular, no se estaría en rigor de verdad frente a un hecho imprevisible ni siempre ajeno. Aunque tenga o adquiera entidad catastrófica para el ser humano, ha sido largamente anunciada y las responsabilidades parecen estar cada vez más claras. Dilemas similares enfrenta la definición de una situación de emergencia ambiental.

\footnotetext{
18 Vid. AGAMBEN, Giorgio (2007). Estado de Excepción, Buenos Aires, AH; GUIBOURG, A. Ricardo (2003) (Dir.), Emergencia económica y teoría del derecho, Buenos Aires, La Ley; GONZALEZ MORAS (2002), J. M., “Estado de excepción. Soberanía, Poder de Policía y Emergencia", en La radicalidad de las formas jurídicas. Críticas a la razón cínica, AA.VV., Ediciones Grupo La Grieta, Buenos Aires.
} 
Siendo previsible que los eventos extremos o catastróficos se incrementen con carácter progresivo, los ordenamientos jurídicos deberán ir adaptándose para dar rápida respuesta a desafíos que, cuando desborden el marco de la actuación preventiva si la hubiere, adquirirán natural y nuevamente la forma de emergencia. Incluso los ordenamientos jurídicos que han iniciado o consolidado un cambio de paradigma hacia normas y políticas de tipo preventivo en vez del predominante reactivo, pueden seguir expuestas a los dilemas que plantea la propia definición y utilización de la emergencia antes referida.

Aquí aparece la cuestión capital de los denominados nuevos normales, es decir, los nuevos parámetros o patrones que comienzan a perfilarse a partir de un cambio climático avanzado que torna rápidamente obsoleto lo considerado "normal" para un tiempo pasado, a la vez que desborda las reglas e instrumentos para su manejo. ¿Será considerada una situación de emergencia climática sin retorno el aumento promedio en la temperatura global de $1,5 \mathrm{C}^{\circ}$ ? Lo será de $2 \mathrm{C}^{\circ}$ ? O será preciso establecer un nuevo normal para ese umbral? Esta pregunta nos lleva al segundo elemento fundamental para configurar la situación de emergencia.

\section{Duración o plazo de la emergencia}

Este elemento resulta crucial para definir la afectación a los principios republicanos y democráticos habida cuenta que si la emergencia no reviste carácter temporal como lo exige la doctrina y jurisprudencia acaba por transformarse en permanente. Parece lógico que la emergencia se extienda mientras se mantengan las causas que determinaron su declaración, lo que puede resultar más o menos claro en el caso de la emergencia hídrica, menos claro en la ambiental y muy difuso en materia climática si se toma, por ejemplo, el aumento de la temperatura calificado como de no retorno, como criterio.

Como el mantenimiento indefinido de la emergencia compromete el estado de derecho, el problema se traslada nuevamente 
a la definición misma de las condiciones y criterios objetivos utilizados para determinarla antes referidos, que de otra forma deberán ser revisados para el establecimiento de esos nuevos normales. De manera tal que existe una relación excluyente entre la emergencia, como excepción al ordenamiento jurídico y su duración. Si las condiciones tenidas en cuenta para declararla se mantienen, o se revisa la definición o se discontinúa la emergencia. Y ello lleva al tercer elemento.

El aumento en la frecuencia de uso de declaraciones de emergencia suspendiendo la aplicación de la ley y habilitando competencias extraordinarias para la autoridad, cuya reiteración o prórroga extiende o perpetúa el período de excepción, acentúa el fenómeno e invierte el orden entre norma de principio y excepción, estructurante del fenómeno jurídico.

Sin embargo, la configuración de un estado de emergencia o excepción permanente no se presenta de manera lineal. Una situación de este tipo podría plantearse, por ejemplo, con más de diez años consecutivos de declaraciones de emergencia por sequía, donde la razonabilidad y sentido de la medida empieza a ponerse en duda, tal como ha ocurrido en materia económica en múltiples oportunidades.

La prolongación indefinida de la emergencia puede darse de manera explícita, visible, pero también, implícita o difusa. Así, para la potencial configuración de una situación de emergencia permanente o estado de excepción no hace falta estar bajo una declaración formal permanente de emergencia, sino que basta con que con cierta frecuencia se habilite una emergencia que permita tomar una serie de decisiones que proyectan sus efectos o consecuencias más allá de la misma, como para que también pueda considerarse configurado ese estado.

\section{Conclusiones}

La emergencia, desde la óptica de la excepción, como justificación de la suspensión del ordenamiento jurídico y atribución de facultades extraordinarias a los poderes públicos con las 
características indicadas en el caso de la emergencia hídrica, no parece la mejor respuesta a los desafíos que plantea la cuestión ambiental y el cambio climático al derecho en el siglo XXI.

Sin embargo, lo dicho hasta aquí lleva a la convicción de que la emergencia (tanto real como producida o inducida) será el contexto cada vez más frecuente de actuación de los poderes públicos en materia hídrica, ambiental y, desde luego, climática, de donde distinguirlas y tematizarlas debidamente deviene crucial.

El estudio del fenómeno de la emergencia en estos ámbitos lleva a replantear la dinámica de funcionamiento del derecho mismo, que no es otra cosa que las normas prestablecidas para una determinada normalidad climática que está cambiando rápidamente junto con el ambiente y la disponibilidad de recursos hídricos hasta ahora considerada normal.

De allí, que resulte relevante comprender cómo una emergencia hídrica o ambiental, como excepción al ordenamiento jurídico, aunque diferente desde la óptica jurídica, no resulta completamente desconectada de la emergencia climática que, de contenido simbólico o discursivo, es proclamada con amplia generosidad por parlamentos y funcionarios como contrapartida. Y esto es así porque el etéreo ámbito del cambio climático carece de una materialidad propia, autónoma, que sólo adquiere a través del ambiente y los recursos hídricos donde se manifiestan sus consecuencias.

Esto significa que si bien la emergencia climática se mueve por el momento en un plano meramente discursivo, no ocurre lo mismo con los ámbitos donde se materializan sus efectos, como los recursos hídricos, con sequías e inundaciones, por ejemplo, donde el incremento del uso de dispositivos de emergencia de contenido excepcionador parece, en cambio, evidente. Además de proyectar sus efectos concretos a través de esos ámbitos, la emergencia climática declamada comienza a proveer de justificación adicional, a las emergencias hídricas y ambientales, que a diferencia del carácter de la primera, sí inciden en 
el orden jurídico disponiendo muchas veces la excepción de las normas prestablecidas para lidiar con sus consecuencias.

Con ello, repensar el derecho de aguas, ambiental y climático desde los presupuestos y la óptica de la emergencia puede contribuir a mejorar la calidad de los marcos normativos y las decisiones urgentes de gobierno manteniendo la compatibilidad con los presupuestos constitucionales del estado de derecho.

En ese sentido, pueden profundizarse los marcos legales de esa actividad de emergencia, las condiciones y criterios objetivos a considerar para su procedencia y duración, debe especificarse el alcance de las facultades y prerrogativas de excepción en cada caso y los órganos encargados de llevarlas a cabo. Debe asimismo asegurarse la inmediata rendición de cuentas y el contralor ex post de las medidas adoptadas y los fondos invertidos de urgencia al margen de los procedimientos normales de adjudicación y contratación. También deben construirse estándares adecuados de control judicial suficiente tanto para la declaración misma que la justifica, como para la actuación que, como consecuencia de ella, tenga lugar.

Sin embargo, no todos los países tienen las mismas capacidades y posibilidades para adoptar efectivamente paradigmas preventivos de gestión de riesgos, políticas y regulaciones que minimicen el recurso a dispositivos de emergencia. No todos, tampoco, tienen un estado de derecho que comprometer. Aun así, el recurso a la emergencia como dispositivo de gobierno que suspende o interrumpe la aplicación de la ley, no depende solamente de la implementación de los instrumentos adecuados, sino también de la responsabilidad política de quienes ejercen el poder y la fortaleza institucional y social para limitar su abuso.

\section{Bibliografía}

AAVV (2009), Agua, Territorio, Cambio Climático y derecho Administrativo, Monografías de la Revista Aragonesa de Administración Pública, Zaragoza, Numero Extraordinario XI.

AGAMBEN, Giorgio (2007), Estado de Excepción, Buenos Aires, AH. 
BOTTERILL, Linda Courtenay, HAYES, Michael J., (2012), « Drought triggers and declarations: science and policy considerations for drought risk management ", Natural Hazards (2012) 146.

BRUFAO CURIEL, Pedro (2012). El régimen jurídico de las sequías: crítica a la regulación extraordinaria y urgente de un fenómeno natural y cíclico propio del clima, RAP, 187, pp. 199-239.

CRAIG, Robin Kundis (2010), Adapting Water Law to Public Necessity: Reframing Climate Change Adaptation as Emergency Response and Preparedness (September 17, 2010). Vermont Journal of Environmental Law, Vol. 11, No 4, pp. 709-756.

EMBID IRUJO, Antonio (Dir.) (2018), Sequía e inundación como fenómenos hidrológicos extremos, Cizur Menor, Thomson Reuters Aranzadi, 492 pp.

EMBID IRUJO, Antonio; MARTÍN, Liber (2017), El nexo entre el agua, la energía y la alimentación en América Latina y el Caribe. Planificación, marco normativo e identificación de interconexiones prioritarias. SRNI, Santiago, CEPAL, $70 \mathrm{pp}$.

GONZALEZ MORAS (2002), J. M., "Estado de excepción. Soberanía, Poder de Policía y Emergencia”, en La radicalidad de las formas jurídicas. Críticas a la razón cínica, AA.VV., Ediciones Grupo La Grieta, Buenos Aires.

GREEN, Micah (2014). Keeping California's Thirst for Groundwater in Check, in McGeorge Law Review, Volume 46-2, pp. 346 y 347.

GUIBOURG, A. Ricardo (2003) (Dir.), Emergencia económica y teoría del derecho, Buenos Aires, La Ley.

HALPÉRIN, Jean-Louis; MILLARD, Eric; VAUCHEZ, Stéphanie H. (2017), L'état d'urgence: de l'exception à la banalisation, Paris, PUPN, 268 pp.

HANAK, Ellen et al. (2014): Modernizing drought water allocations. http://californiawaterblog.com/2014/10/16/modernizingdrought-water-allocations/

HOWE, Charles (2008). Water Law and Economics: An Assessment of River Calls and the South Platte Well Shut-Down, 12 U. Denv. Water L. Rev. 181 (2008-2009).

LINDSAY, Bruce (2010), Climate of exception: what might a "climate emergency" mean in law?, Federal Law Review, Volume 38, pp 255-281. https://doi.org/10.22145/flr.38.2.4 
LUND, Jay; LORD, Ben; FLEENOR, William y WILLIS, Ann. (2014): Drought Curtailment of Water Rights - Problems and Technical Solutions. Center for Watershed Sciences, UC Davis. Technical comments to the State Water Resources Control Board.

MARTIN Liber (2010), Derecho de Aguas. Estudio sobre el uso y dominio de las aguas públicas, Buenos Aires, Abeledo Perrot.

MARTÍN Liber y PINTO Mauricio (2015). Escasez extraordinaria y derecho de aguas en: Revista de Derecho Administrativo Económico $\mathrm{N}^{\mathrm{o}} 20$, Santiago, PUCC.

ROSSI, Giuseppe y CANCELLIERE, Antonio (2013). Managing drought risk in water supply systems in Europe: in Journal of Water Resources Development, 29:2, pp. 272-289, DOI: 10.1080/07900627.2012.713848.

SALINAS ALCEGA, Sergio (2014), El cambio climático: Entre cooperación y conflicto. Propuestas desde el derecho internacional, $285 \mathrm{pp}$.

NOÉ SCHEINWALD, Paula (2015). Interfaz ciencia-política en la gestión de sequía en Chile, Academia.edu

URQUIJO, Julia, et al, (2005) Drought and exceptional laws in Spain: the official water discourse, en International Environmental Agreements. Politics. Law and Economics, Springer.

VERGARA BLANCO, Alejandro (1999). El derecho de la sequía: la redistribución de aguas, en Revista de Derecho Administrativo Económico, Vol. I / No 2, pp. 361-370. 